\title{
Analysis of Behavioral Selection After Sensory Deprivation of Legs in the Cricket Gryllus bimaculatus
}

\author{
Masamichi Kanou $^{1 *}$, Shinsuke Morita ${ }^{1 \ddagger}$, Tetsuya Matsuura $^{1 \dagger}$ \\ and Tsuneo Yamaguchi ${ }^{2}$ \\ ${ }^{1}$ Department of Biology, Graduate School of Science and Engineering, Ehime University, \\ Matsuyama 790-8577, Japan \\ ${ }^{2}$ Kawasaki College of Allied Health Profession, Kurashiki, Okayama 701-0194, Japan
}

\begin{abstract}
An air puff stimulus evoked the swimming of an intact cricket, Gryllus bimaculatus, placed on a water surface. When only the forelegs were intact, swimming was initiated frequently, but flying was never initiated. On the other hand, flying was initiated when only the middle legs or hindlegs were intact. Therefore, the sensory inputs from the forelegs are important in the initiation of swimming and for the inhibition of flying when on the water surface. After bilateral ablation of the middle legs and hindlegs, the bilateral segments of the remaining forelegs were sequentially ablated from the distal area to the proximal area of the legs. After bilateral ablation of all tarsomeres, the relative occurrence of swimming decreased and that of flying increased. After the following ablation of the bilateral tibiae, most insects responded to an air puff stimulus by flying. Experiments performed after coating the leg surface with enamel resulted in almost the same behavioral change as that observed in the ablation experiments. These results suggest that the sensory receptors responsible for the initiation of swimming and the inhibition of flying are mainly located on the surface of the tibia and the tarsus of the forelegs. The behavioral change between swimming and walking was also studied using methylcellulose solutions of various viscosities. On the methylcellulose solution, the relative occurrence of walking in the crickets increased with an increase in the viscosity of the solution.
\end{abstract}

Key words: cricket, behavioral selection, water receptor, swimming, flying

\section{INTRODUCTION}

From a wide range of behaviors, animals have to select the one most appropriate in a particular situation. To operate an adequate motor program, many types of sensory system must be recruited. Such a selection of behaviors must frequently occur in all animals so the animals can survive in ever-changing natural environments.

Crickets bear a pair of appendages called cerci at the end of the abdomen. There are a large number of mechanosensitive filiform hairs on the cerci. Information on air motion around a cricket is detected by these filiform hairs (Bentley, 1975; Shimozawa and Kanou, 1984a, b) and is sent to the terminal abdominal ganglion (TAG). In TAG, giant interneurons (GIs) integrate the information and send it to the other parts of the body (Murphey et al., 1977; Kanou and Shimozawa, 1984). This information occasionally elicits an escape behavior (running or jumping) when the cricket is placed on the ground (Gras and Hörner, 1992; Tauber and Camhi, 1995; Kanou et al., 1999, 2001, 2002; Kanou and Kondoh, 2004). However, an identical air puff evokes differ-

\footnotetext{
* Corresponding author. Phone: +81-89-927-9631;

Fax : +81-89-927-9631;

E-mail: kanou@mserv.sci.ehime-u.ac.jp

† Present address: Department of Welfare Engineering, Faculty of Engineering, Iwate University, Morioka 020-8551, Japan

₹ The first two authors contributed equally to this work. doi: $10.2108 / z$ sj.24.945
}

ent behaviors depending on the situation of the cricket, that is, flying when it is suspended in the air (Tomioka and Yamaguchi, 1980) and swimming when it is placed on a water surface (Matsuura et al., 2002).

The wind-evoked behaviors of crickets mentioned above consist of rhythmically repeated motions of the legs or wings. Therefore, a particular pattern generator (PG) (e.g., Wilson, 1961) for a particular locomotion must start to operate when the cricket is responding to an identical air puff applied to the cerci. Our question was how a particular PG is selected under a particular environmental condition. In the behavioral selection by the crickets mentioned above, sensory inputs particularly from the legs likely play a significant role, because, when placed on a water surface, a cricket flies in response to an air puff when all six legs are ablated (Matsuura et al., 2002). To determine the distribution of the receptors controlling the behavioral change between swimming and flying, ablation and surface coating of leg segments were carried out. On the basis of the relative occurrences of wind-evoked behaviors in these crickets, the distribution of receptors essential for the selection was estimated. In addition, although preliminary, the behavioral change between swimming and walking was also studied by changing the viscosity of the water substrate.

Morphological and electrophysiological investigations of the receptors controlling the behavioral selection are reported in an accompanying paper (Kanou et al., 2007). 


\section{MATERIALS AND METHODS}

\section{Animals}

Adult female crickets, Gryllus bimaculatus, were used in the experiments. In the behavioral experiments, the crickets were used one day after the final molt, because their flight muscles start to degenerate from almost two days after the molt (Shiga et al., 1991). The crickets were reared in our laboratory and fed with insect chow (Oriental Kohbo) and carrots. The temperature of the culture room was in the range of $28-30^{\circ} \mathrm{C}$ and the LD cycle was $12: 12$. The details of the cricket rearing are the same as those in previous papers (Kanou et al., 1999; Matsuura et al., 2002).

\section{Sensory deprivation}

Sensory deprivation of the legs was carried out by using the following two methods. In the first method, a leg segment or leg segments were cut with a razor blade or a pair of small scissors under low-temperature anesthesia. Because the amount of body fluid lost from the wound was very small, the bleeding did not have any influence on the elicited behavior. Experiments were initiated after the body fluid coagulated at the wound site. In the second method, a leg segment or leg segments were coated with enamel (nail polish). During the coating, special attention was paid not to fix any leg joints.

\section{Behavioral analysis}

A cricket was placed on a water surface by supporting the body with a metal rod (Fig. 1). One end of the metal rod was fixed to the sternum of the thorax and the other end was fixed to a micromanipulator for adjusting the cricket's position to simulate floating on the water surface. In this position, almost half of the body (ventral side), including all six legs, was in the water but the dorsal side of the body was above water. In non-tethered adult crickets, the ventral side of cerci sometimes touched the water surface. In the tethered crickets, however, the body was pitched forward slightly to prevent the cerci from getting wet. When a tethered cricket was placed on the water, it started to swim spontaneously, but the swimming stopped after a while. Experiments were started after the spontaneous swimming stopped. All wings were cut at approximately onethird of the way up from the stumps, because the surface tension of water sometimes prevents the wings from moving smoothly.

The stimulus air puff was a jet of air produced by an air compressor (Bebicon; Hitachi). The nozzle used for delivering the air puff was set $18 \mathrm{~cm}$ away from the cricket and 15 degrees above the horizontal plane (Fig. 1). The velocity of the air was controlled by adjusting the flow volume per unit of time by using a flow controller

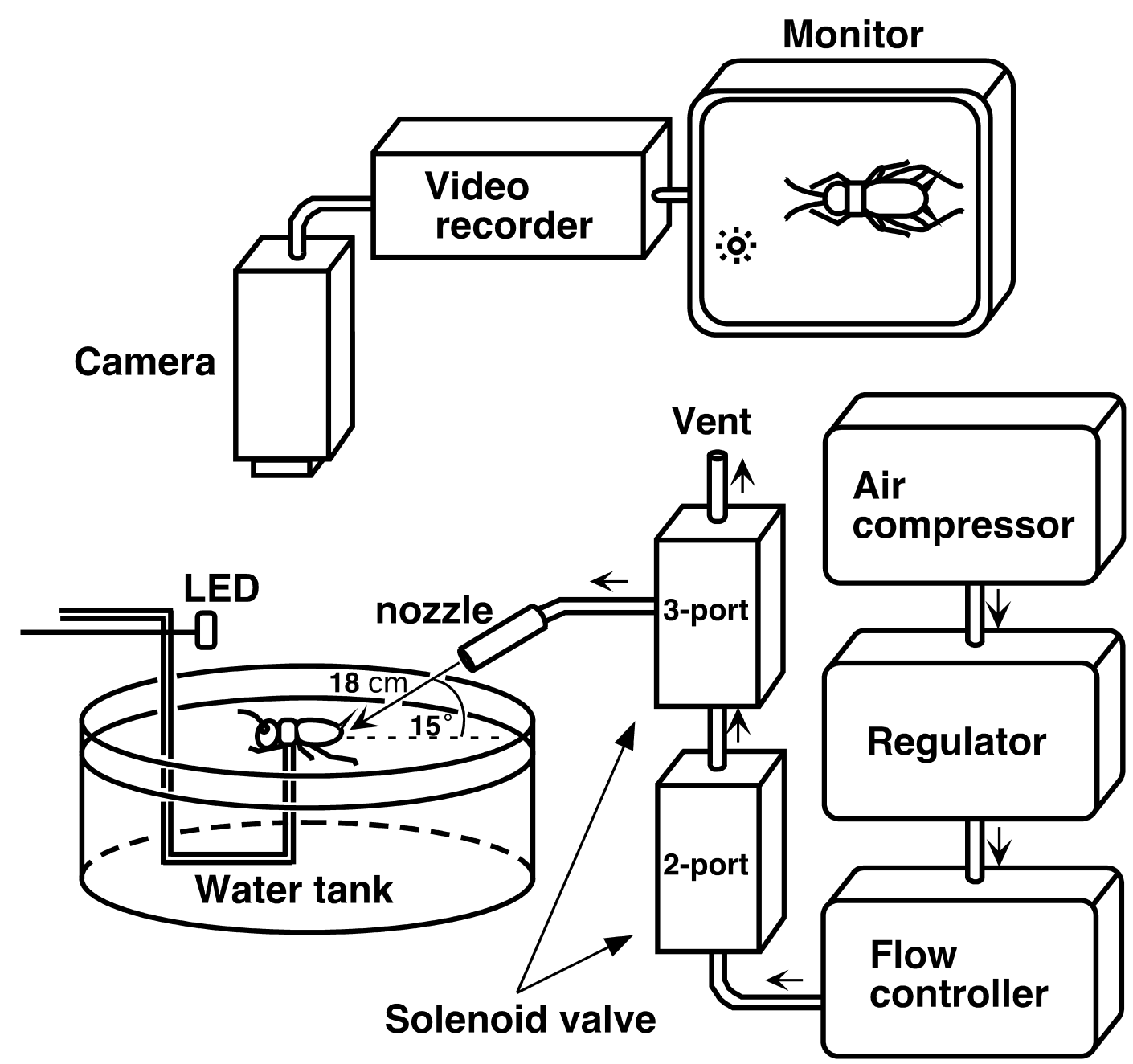

Fig. 1. Experimental setup to study wind-evoked behavior on water or on a methylcellulose (MC) solution. A cricket was supported by a metal rod to simulate floating on the surface. The stimulus air puff was a jet of compressed air and was delivered from a nozzle set $18 \mathrm{~cm}$ away from the insect. Behaviors were monitored and recorded using a video camera set immediately above the insect. See text for details. 
(2504FR, Koflok). The peak velocity of the air at the cricket cerci was $3.9 \mathrm{~m} / \mathrm{sec}$, and the stimulus duration was $100 \mathrm{msec}$, because such stimulus is sufficient to elicit the swimming of a cricket (Matsuura et al., 2002). The details of the air-puff stimulation were the same as those in our previous studies (Kanou et al., 1999, 2002; Matsuura et al., 2002).

All behaviors elicited by the air puff were recorded with a video camera (DXC-151, SONY) connected to a recorder (DSR-30, SONY), and later analyzed by viewing them on a CRT screen. An LED set near the cricket (Fig. 1) was synchronously lit at the start of the air puff to specify the timing of the stimulation during the off-line analysis.

\section{RESULTS}

\section{Wind-evoked behaviors of crickets on a water surface}

Nontethered intact crickets placed on the water surface usually started swimming spontaneously but stopped after a while. In response to an air-puff stimulus applied to the cerci, the crickets started swimming again (Matsuura et al., 2002). Differently from the leg motion during tripod walking (Gray, 1968; Matsuura et al., 2002), each pair of legs moved synchronously during swimming. The leg motion shifted in the order of the forelegs, middle legs and hindlegs, and returned to the forelegs to start next cycle. These behavioral patterns on the water were also observed in the tethered crickets used in this study.

In this study, the elicited behavior was assessed as "swimming" when more than two such cycles of leg motion were observed. However, because other behaviors were also observed in response to an air puff, we classified the wind-evoked behaviors on the water surface as follows. When a cricket made an irregular leg motion in response to an air puff, it was called St (struggle). Sometimes a cricket extended all of its legs in response to the stimulation and maintained this posture for a while. Such behavior was called ExL (extension of the legs). When swimming was elicited immediately after St or ExL, it was also assessed as "swimming". In addition to these behaviors, walking was occasionally elicited on the water surface depending on the experimental situation.

In nine groups of intact crickets, wind-evoked behaviors were studied when the crickets were placed on the water surface (Table 1). Each group contained 20 crickets, and 10 stimuli were applied to each cricket. Therefore, the total number of trials in one group was 200 and that for the nine groups was 1,800 . An interval of more than one minute was inserted between sequential trials for each cricket. Swimming was observed in $47.1 \pm 8.2 \%$ (mean $\pm S D$ ) of the trials, and the rest showed St and ExL. Flying was observed in
Table 1. Wind-evoked behaviors of crickets placed on water surface (\%)

\begin{tabular}{ccrccc}
\hline Group & Swimming & \multicolumn{1}{c}{ St } & ExL & Flying & $\mathrm{N}$ \\
\hline 1 & 45.5 & 4.0 & 50.5 & 0.0 & 20 \\
2 & 50.0 & 11.0 & 39.0 & 0.0 & 20 \\
3 & 41.5 & 13.0 & 45.5 & 0.0 & 20 \\
4 & 31.5 & 25.5 & 42.5 & 0.5 & 20 \\
5 & 52.5 & 9.0 & 38.5 & 0.0 & 20 \\
6 & 47.5 & 9.0 & 43.5 & 0.0 & 20 \\
7 & 42.5 & 9.0 & 49.0 & 0.0 & 20 \\
8 & 59.1 & 7.3 & 33.6 & 0.0 & 20 \\
9 & 54.5 & 8.0 & 37.5 & 0.0 & 20 \\
\hline Mean & 47.1 & 10.6 & 42.2 & 0.1 & \\
SD & 8.2 & 6.1 & 5.6 & 0.2 & \\
\hline
\end{tabular}

The results of 10 trials per animal. $\mathrm{N}=$ number of crickets used.

only one of the 1,800 trials.

\section{Effects of ablation of legs or leg segments on selection of wind-evoked behaviors}

Our question was whether all the legs are necessary or whether a particular part of a particular leg is sufficient for the behavioral selection. To explore this, we first made bilateral ablations of a particular pair of legs at their coxal segments. An air puff was presented to the operated crickets placed on the water surface, and the relative occurrences of the elicited behaviors were determined (Table 2).

Initiation of swimming

When the forelegs were ablated, the relative occurrence of swimming showed a significant decrease from $45.5 \%$ to $30.0 \%(P<0.01)$. On the other hand, flying, which was not observed before the ablation, was observed in $7.0 \%$ of the trials. The relative occurrence of St showed a significant increase from $4.0 \%$ to $33.0 \% \quad(P<0.01)$ and that of $\mathrm{ExL}$ showed a significant decrease from $50.5 \%$ to $30.0 \%$ $(P<0.01)$. After the ablation of the middle legs or hindlegs, the relative occurrences of swimming, St and ExL showed only small changes, and none of them was significant (Table 2). These results suggest that the initiation of swimming on the water surface largely depends on sensory information from the forelegs.

To determine which part or parts of the three pairs of legs are essential for the behavioral selection, two pairs of legs were bilaterally ablated and only one pair of legs was left. The leg segments of the remaining legs were then sequentially ablated from the distal area to the proximal area, and the relative occurrences of wind-evoked behaviors

Table 2. Changes in wind-evoked behaviors after bilateral ablation of legs (\%)

\begin{tabular}{lrrccc}
\hline & Swimming & \multicolumn{1}{c}{ St } & ExL & Flying & $\mathrm{N}$ \\
\hline Intact & $45.5 \pm 22.1$ & $4.0 \pm 6.0$ & $50.5 \pm 20.6$ & 0.0 & 20 \\
Foreleg ablation & ${ }^{* *} 30.0 \pm 24.1$ & ${ }^{* *} 33.0 \pm 29.2$ & ${ }^{* *} 30.0 \pm 24.5$ & ${ }^{*} 7.0 \pm 13.8$ & 20 \\
& & & & & \\
Intact & $50.0 \pm 24.7$ & $11.0 \pm 13.7$ & $39.0 \pm 22.9$ & 0.0 & 20 \\
Middle leg ablation & $42.9 \pm 29.8$ & $13.0 \pm 23.6$ & $44.1 \pm 30.1$ & 0.0 & 20 \\
Intact & $41.5 \pm 24.3$ & $13.0 \pm 14.9$ & $45.5 \pm 24.1$ & 0.0 & 20 \\
Hindleg ablation & $34.0 \pm 29.8$ & $18.0 \pm 21.7$ & $48.0 \pm 25.4$ & 0.0 & 20 \\
\hline
\end{tabular}

The results of 10 trials per animal. $\mathrm{N}=$ number of crickets used.

Asterisk: significantly different from those for intact crickets. ${ }^{\star}(P<0.05) .{ }^{\star \star}(P<0.01)$. 
were investigated (Fig. 2). When only the forelegs remained, after ablation of the middle legs and hindlegs, the relative occurrence of swimming in the intact crickets $(52.5 \%)$ showed a significant decrease to $33.0 \%(P<0.05$; Fig. $2 \mathrm{~A})$. In the same manner, the relative occurrence of swimming showed significant decreases from $47.5 \%$ to $16.0 \%$ $(P<0.01)$ and from $42.0 \%$ to $10.5 \%(P<0.01)$ in crickets with only the middle legs (Fig. 2B) and hindlegs (Fig. 2C) intact, respectively. These results suggest that swimming can be initiated when any one pair of legs is intact, but that the sensory inputs from the forelegs play a more significant role in initiating swimming than those from the other legs.

Next, particular leg segments were ablated and the changes in the relative occurrences of wind-evoked behaviors were investigated. Because a tarsus consists of four tarsomeres (the most distal tarsomere is a pair of claws), at first the two distal tarsomeres were bilaterally ablated from each remaining leg. The relative occurrences of swimming after the ablation showed significant decreases, that is, $7.0 \%, 4.5 \%$ and $5.0 \%$ in crickets with only the forelegs, middle legs and hindlegs remaining, respectively (Claws ab. in Fig. 2A-C). After the subsequent ablation of the two remaining tarsomeres, the relative occurrences of swimming were $4.5 \%, 1.5 \%$ and $3.5 \%$ in crickets with only the forelegs, middle legs and hindlegs remaining, respectively (Tarsi ab. in Fig. 2A-C). Therefore, in every case, swimming was observed only in a small number of crickets after ablation of all the tarsomeres. After bilateral ablation of the tibial segments, no swimming was observed (Tibiae ab. in Fig. 2AC).

\section{Initiation of flying}

Although intact crickets hardly showed flying behaviors on the water surface, the relative occurrence of flying increased after leg ablation. The relative occurrences of flying with only the forelegs, middle legs and hindlegs intact were $3.5 \%, 24.5 \%$ and $9.0 \%$, respectively (Fig. $2 \mathrm{~A}-\mathrm{C}$ ).
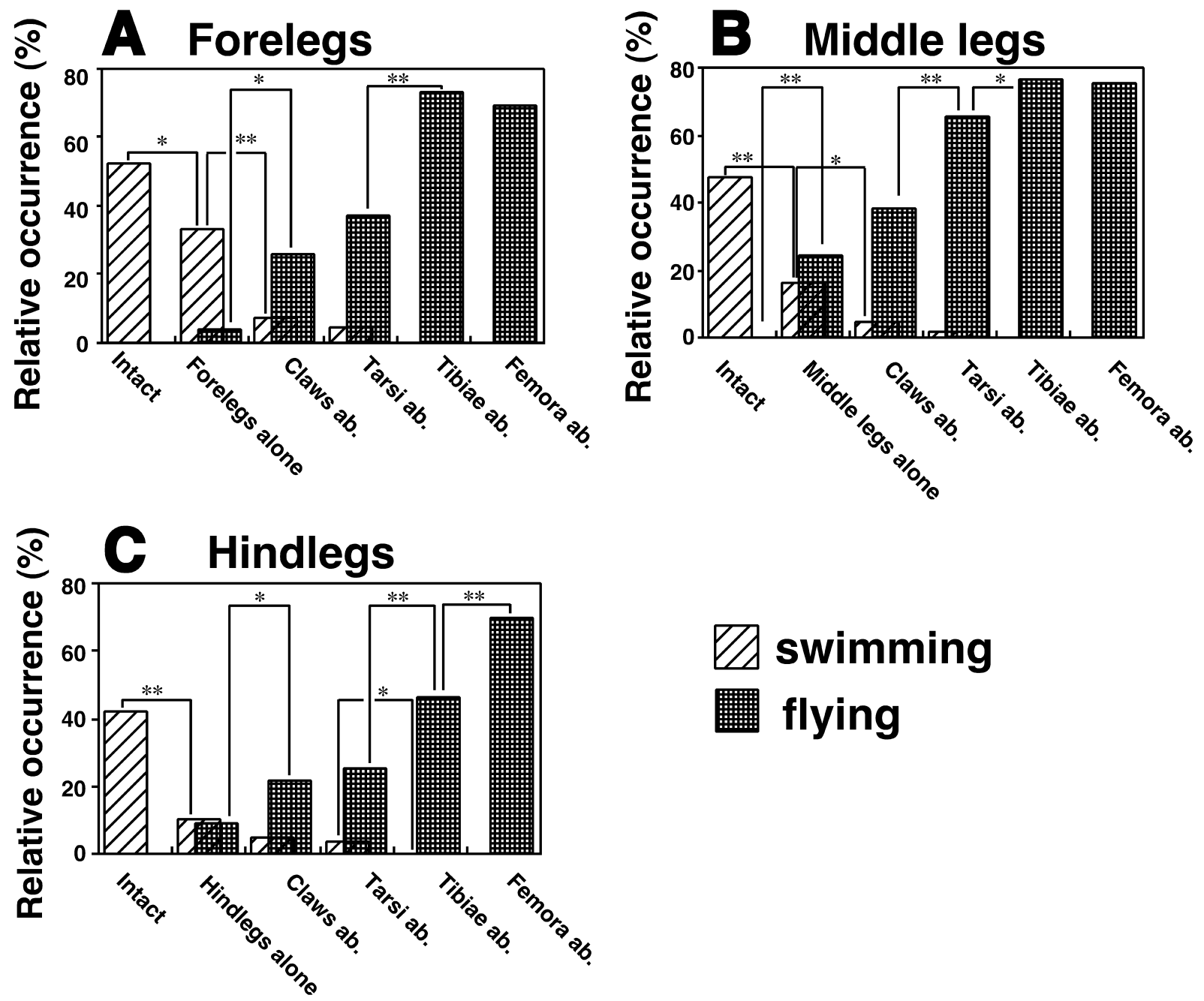

Fig. 2. Effects of sequential ablation of leg segments on the relative occurrences of wind-evoked behaviors when a cricket was placed on a water surface. Two pairs of legs were ablated, leaving only the forelegs (A), middle legs (B) or hindlegs (C). Then, two of the distal tarsomeres (Claws ab.), the remaining two tarsomeres (Tarsi ab.), tibial segments (Tibiae ab.), and femoral segments (Femora ab.) of the remaining legs were sequentially ablated, and the relative occurrences of swimming and flying were noted. In every experiment $(A, B$ and $C)$, the relative occurrence of swimming decreased and that of flying increased with the progression of ablation of the leg segments. Note that the relative occurrence of swimming was still high when the forelegs were intact. Statistically significant differences were determined; ${ }^{*}(P<0.05)$, ${ }^{\star *}(P<0.01)$. See text for details. 
Thus, flying is strongly suppressed by sensory inputs from the forelegs.

The relative occurrence of flying was monitored during the sequential ablation of the remaining leg segments (Fig. $2 A-C)$. In crickets with only the forelegs or middle legs remaining, the relative occurrence of flying increased until the ablation of the tibial segments, but did not show any change after the ablation of the femoral segments (Fig. 2A$\mathrm{B})$. In crickets with only the hindlegs remaining, on the other hand, the relative occurrence of flying was lower than that in crickets with only the forelegs or middle legs remaining and increased even after ablation of the femoral segments (Fig. 2C).

\section{Effects of leg surface coating on behavioral selection}

Sequential ablation of the leg segments revealed the distribution of unknown sensory organs essential for behavioral selection. However, this ablation might have destroyed some internal sensory organs, e.g., the joint chordotonal organs of the legs. To test whether this occurred, we coated the leg surface with enamel in crickets with only the forelegs intact (both the middle legs and hindlegs were ablated).

The relative occurrences of swimming, St, ExL and flying in a group of intact crickets placed on the water surface were 59.1, 7.3, 33.6 and $0 \%$, respectively (Table 3). Sequential ablation or coating of the leg segments was carried out on these crickets as follows. First, both the middle legs and hindlegs of the crickets were bilaterally ablated. After the ablation, the relative occurrences of swimming, St, ExL and flying were $38.7,17.7,36.8$ and $6.8 \%$, respectively. Second, all the tarsomeres of the forelegs were bilaterally coated. After the coating, the relative occurrence of swimming showed a significant decrease to $8.2 \%(P<0.01)$ and that of flying showed a significant increase to $24.1 \%$ $(P<0.01)$. Third, tibial segments were bilaterally coated. After the coating, the relative occurrence of swimming again showed a significant decrease to $2.7 \%(P<0.05)$ and that of flying showed a significant increase to $49.5 \% \quad(P<0.01)$. Fourth, femoral segments were bilaterally coated. The coating showed no effect on the relative occurrence of any behavior (Table 3 ). The results of this sequential sensory deprivation by coating were almost identical with the results of the ablation experiments (Fig. 2A). The results obtained in this study suggest that the sensory organs essential for the behavioral change between swimming and flying have some structures on the leg surface.

In crickets with only the forelegs intact, the relative occurrences of wind-evoked behaviors were determined using different sequences of coating (Table 4). In a group of intact crickets investigated, the relative occurrences of swimming, St, ExL and flying were 55.3, 7.6, 37.1 and $0 \%$, respectively. After bilateral ablation of both the middle legs and hindlegs of the crickets, the relative occurrences of swimming, St, ExL and flying were 35.3, 7.6, 54.7 and 2.4\%, respectively. After coating the tibial surface of the forelegs, the relative occurrence of swimming decreased significantly $(18.8 \% ; P<0.05)$ and that of flying increased to $13.5 \%$ (not significant). After coating all the tarsomeres, the relative occurrence of swimming again decreased significantly $(0.6 \% ; P<0.05)$ and that of flying increased significantly (32.4\%; $P<0.05)$. These results were compared with those of the experiment in which leg segments were coated using a different sequence (Table 3 ). When the tibial segments were coated first, the relative occurrence decreased by $16.5 \%$ (from $35.3 \%$ to $18.8 \%$ ) for swimming and the relative occurrence increased by $11.1 \%$ (from $2.4 \%$ to $13.5 \%$ ) for flying (Table 4). On the other hand, when the tarsomeres were coated first, the relative occurrence decreased by $30.5 \%$ (from $38.7 \%$ to $8.2 \%$ ) for swimming and the relative occurrence increased by $17.3 \%$ (from $6.8 \%$ to $24.1 \%$ ) for flying (Table 3). These results suggest that, even though the coating of the tarsal and tibial segments caused a decrease in

Table 3. Effects of sensory deprivation of foreleg segments (\%)

\begin{tabular}{lcccc}
\hline & Swimming & St & ExL & Flying \\
\hline Intact & 59.1 & 7.3 & 33.6 & 0.0 \\
Middle and hindlegs ablation & $* \star 38.7$ & 17.7 & 36.8 & 6.8 \\
Tarsi coating & ${ }^{* *} 8.2$ & 19.5 & 48.2 & ${ }^{* *} 24.1$ \\
Tibiae coating & $* 2.7$ & 16.4 & 31.4 & ${ }^{* *} 49.5$ \\
Femur coating & 2.4 & 10 & 38.2 & 49.4 \\
\hline
\end{tabular}

20 crickets were used. The results of 10 trials per animal.

Legs were sequentially ablated or coated from upper to lower.

Asterisk: significantly different from those for previous states. ${ }^{*}(P<0.05) .{ }^{* \star}(P<0.01)$.

Table 4. Effects of sensory deprivation of foreleg segments (\%)

\begin{tabular}{lccrr}
\hline & Swimming & St & ExL & Flying \\
\hline Intact & 55.3 & 7.6 & 37.1 & 0.0 \\
Middle and hindlegs ablation & ${ }^{*} 35.3$ & 7.6 & ${ }^{*} 54.7$ & 2.4 \\
Tibiae coating & ${ }^{*} 18.8$ & ${ }^{*} 19.4$ & 48.3 & 13.5 \\
Tarsi coating & ${ }^{*} 0.6$ & 16.5 & 50.5 & ${ }^{*} 32.4$ \\
Femur coating & 3.5 & 15.9 & 41.2 & 39.4 \\
\hline
\end{tabular}

20 crickets were used. The results of 10 trials per animal.

Legs were sequentially ablated or coated from upper to lower.

Asterisk: statistically significant from previous states. ${ }^{\star}(P<0.05)$. 


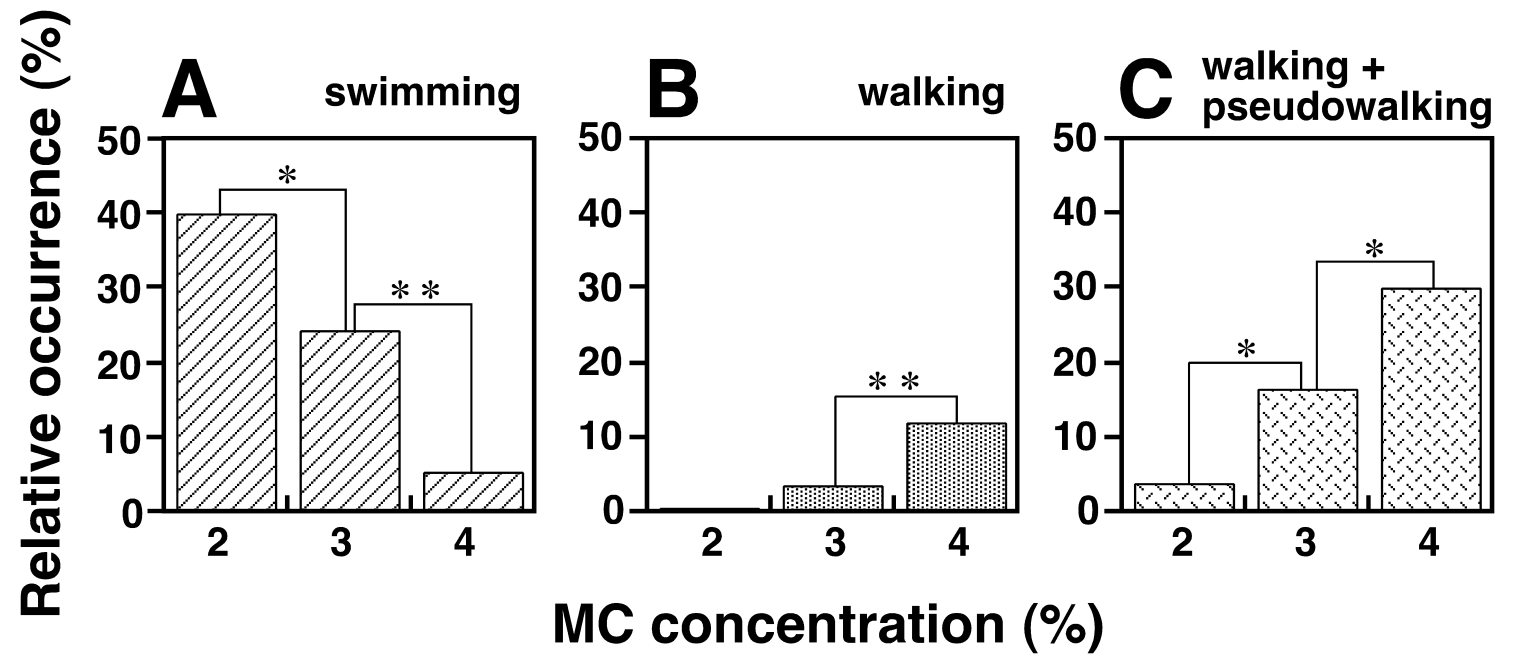

Fig. 3. Relative occurrences of wind-evoked behaviors when a cricket was placed on methylcellulose (MC) solutions of different concentrations (2, 3 and 4\%). (A) Swimming. (B) Walking. (C) Walking + pseudowalking. As the concentration of MC dissolved in distilled water increased, the relative occurrence of swimming decreased and that of walking increased. Statistically significant differences were determined; ${ }^{\star}(P<0.05),{ }^{\star \star}(P<0.01)$. See text for details.

the relative occurrence of swimming and an increase in the relative occurrence of flying, the effect was larger when the tarsal segments were coated.

\section{Behavioral change between swimming and walking}

During the leg-ablation and coating experiments, walking was never observed in crickets placed on the water surface. Leg joints support the body weight and receive frictional resistance from the substrate when the insects are placed on the ground, and these forces are thought to play important roles in triggering walking. To explore this, methylcellulose (MC) solutions at different concentrations (different viscosities) were used instead of the water substrate. The substrates used for the following experiments were 2, 3 and $4 \% \mathrm{MC}$ solutions dissolved in distilled water, with viscosities of 32,364 and 1,344 Pa.sec, respectively. As in the previous experiments, crickets were supported by a metal rod, but in this case were placed on the surface of the $\mathrm{MC}$ solution (Fig. 1). The relative occurrences of swimming in response to an air puff in intact crickets were 40.0, 24.5 and $5.5 \%$ for the 2, 3 and $4 \%$ MC solutions, respectively (Fig. $3 \mathrm{~A})$. Significant differences in the relative occurrences of swimming were observed between the 2 and $3 \% \mathrm{MC}$ solutions $(P<0.05)$ and between the 3 and $4 \% \mathrm{MC}$ solutions $(P<0.01)$. High-viscosity MC solutions thus resulted in a lower relative occurrence of swimming. On the other hand, walking, which was never elicited when crickets were placed on the water, was observed on the $\mathrm{MC}$ solution. The relative occurrences of walking were $0.5,3.5$ and $12.0 \%$ for the 2,3 and $4 \% \mathrm{MC}$ solutions, respectively (Fig. 3B). A significant difference in the relative occurrences of walking was observed between the 3 and $4 \% \mathrm{MC}$ solutions $(P<0.01)$. In contrast to swimming, high-viscosity $\mathrm{MC}$ solutions resulted in a higher relative occurrence of walking. During the experiments, pseudowalking was frequently observed. In pseudowalking, a complete sequence or a part of the sequence of tripod leg motion occasionally appeared during other behaviors such as swimming, St or ExL, but it did not last for more than two cycles. When pseudowalking was counted for walking, the relative occurrences of walking were 4.0, 16.5 and $30.0 \%$ for the 2, 3 and $4 \%$ MC solutions, respectively (Fig. 3C). Significant differences $(P<0.05)$ were observed in the relative occurrences between all the solutions.

\section{DISCUSSION}

Similarly to that in the crickets, it has been reported that identical stimuli elicit different behaviors in the cockroach Periplaneta americana, that is, an air puff evokes escape running when cockroaches are on the ground (Camhi and Tom 1978; Camhi et al., 1978; Tauber and Camhi 1995), whereas the same stimulus initiates flying when they are suspended in the air (Krämer and Markl 1978; Ritzmann et al., 1980). Although we have not yet clarified whether the neural mechanisms for behavioral selection in crickets and that in cockroaches are identical, selection of a particular $P G$ seems to be determined by the number of different sensory inputs in both species.

In a previous study (Matsuura et al., 2002), we showed that sensory inputs from cercal filiform hairs are essential for evoking escape running, swimming or flying, but that other sensory inputs are also necessary to determine which PG for a particular behavior should be selected. In that study, we also noted the possibility that hygroceptors or water receptors on the legs are responsible for transmitting information on sensory inputs for initiating swimming.

\section{Sensory cues to select swimming PG}

Differently from tripod walking (Gray, 1968), each pair of legs of crickets moves synchronously to produce a propelling force during swimming (Matsuura et al., 2002). Other orthopteran insects such as Schistocerca americana and Locusta migratoria show similar leg motions during swimming (Pflüger and Burrows, 1978). However, differently from crickets, the propelling force was produced mainly by the motion of the hindlegs in these insects. In the cricket $G$. 
bimaculatus, the leg-motion pattern for swimming observed in adults is also used by first-stage instars, even when they have had no prior chance to learn how to swim (Matsuura et al., 2002). Therefore, the leg-motion pattern for swimming must be innately programmed in the cricket's CNS as a swimming PG. This swimming PG likely starts operating in response to a particular sensory input or a particular combination of sensory inputs. Four types of swimming-initiating neuron (SIN) that were identified in the suboesophageal ganglion of $G$. bimaculatus (Matsuura et al., 2002) are thought to mediate these sensory inputs and trigger the swimming PG.

In this study, it was found that, in addition to inputs from cercal filiform hairs, inputs from some sensory organs on the legs are necessary for initiating swimming. These sensory organs on the legs have cuticular structures, because the coating experiments (Tables 3,4 ) showed almost the same results as the ablation experiments (Fig. 2). Sensory information from different legs showed different degrees of effectiveness in initiating swimming, and that from the forelegs was more effective than that from other legs (Fig. 2A-C). The sensory information from different leg segments also showed different degrees of effectiveness, with that from the tarsal segment playing a more pivotal role in initiating swimming than that from other segments (Table 3 ). The relative occurrence of swimming gradually decreased with the progression of leg-segment ablation or coating from the distal area to the proximal area. These results suggest that the sensory information used for the initiation of swimming is not from one particular sensory organ, but from a large number of sensory organs scattered across the leg surface. As has been speculated in a previous study (Matsuura et al., 2002), these sensory organs are possibly hygroceptors or water receptors on the leg surface. In the accompanying paper, we report on a morphological and electrophysiological investigation of these organs both (Kanou et al., 2007).

In this study, we revealed that the swimming PG was selected on the basis of information on water transmitted via the legs. However, when a cricket is on the ground, the legmotion pattern of swimming is never elicited, even if a drop of water is applied to the legs. Therefore, the information on water is necessary but is not sufficient to select the swimming PG. This suggests that, in addition to the information on water, some other sensory information must be necessary to select the $P G$.

\section{Flying initiation}

In this study, it was shown that swimming and walking started when particular sensory information was presented. However, the initiation of flying was somewhat different from the initiations of other behaviors. In contrast to swimming, flying occurred increasingly more readily with the progression of leg or leg-segment ablation or coating from the distal area to the proximal area (Fig. 2, Tables 3 and 4). Therefore, unlike other PGs, the flying PG starts to operate when some sensory inputs from the legs are absent, which means that these sensory inputs inhibit flying when a cricket is placed on a water surface or on the ground.

Because coating the leg surface increased the relative occurrence of flying, the sensory organs on the leg surface that inhibit the flying PG must have some structures. How- ever, in addition to the sensory organs located on the leg surface, some unknown sensory organs that are not affected by surface coating seem to provide input inhibitory to the flying PG. When crickets with only the forelegs intact were placed on the water surface, for example, the relative occurrence of flying was almost $70 \%$ after ablation of the tibial segments (Fig. 2A). However, when the tibiae and the more distal leg segments were coated, but the joints were left uncoated to be able to move, the relative occurrence of flying was only $49.5 \%$ (Table 3 ). This result suggests that the unknown sensory organs inhibiting the flying PG are located inside the legs or are not functionally affected by surface coating.

In the cockroach $P$. americana, sensory inputs from hair sensilla ventrally located on the tarsal segments and campaniform sensilla situated on the dorsal surface of the leg segments (except for the coxae) of all six legs prevent the animal from flying (Krämer and Markl, 1978). Although we are not sure whether the system is the same in crickets, a similar mechanism can easily explain the results of the study, because campaniform sensilla, if any, seem to some extent resistant to the effects of enamel coating of the cuticular surface.

\section{Sensory cues for walking initiation}

What is required to initiate the walking PG? Walking was not observed during the leg-ablation or coating experiments. Therefore, unlike the flying PG, the walking PG is not likely to be controlled by inhibitory inputs from sensory organs of the legs. On the other hand, crickets placed on the MC solution were able to walk. The relative occurrence of walking increased with the viscosity of the MC solution (Fig. 3). This suggests that a reacting force from the substrate to the legs is one of the factors important in triggering walking. Therefore, some mechanoreceptors sensitive to such reacting forces probably exist on or inside the legs. Joint chordotonal organs and campaniform sensilla possibly act as such receptors.

\section{ACKNOWLEDGMENTS}

This work was partially supported by grants to M. K. from the Japan Society for the Promotion of Science and the Japanese Ministry of Education, Culture, Sports, Science and Technology.

\section{REFERECNES}

Bentley D (1975) Single gene cricket mutations: effects on behavior, sensilla, sensory neurons, and identified interneurons. Science 187: 760-764

Camhi JM, Tom W (1978) The escape behavior of the cockroach Periplaneta americana. I. Turning response to wind puffs. J Comp Physiol A 128: 193-201

Camhi JM, Tom W, Volman S (1978) The escape behavior of the cockroach Periplaneta americana. II. Detection of natural predators by air displacement. J Comp Physiol A 128: 203-212

Gray J (1968) Animal Locomotion. Weidenfeld and Nicolson, London

Gras H, Hörner M (1992) Wind-evoked escape running of the cricket Gryllus bimaculatus. I. Behavioral analysis. J Exp Biol 171: 189-214

Kanou M, Kondoh Y (2004) Compensation of escape direction in unilaterally cercus-ablated crickets, Gryllus bimaculatus, is associated with the distance walked during recovery period. Zool Sci 21: 933-939 
Kanou M, Shimozawa T (1984) A threshold analysis of cricket cercal interneurons by an alternating air current stimulus. J Comp Physiol A 154: 357-365

Kanou M, Ohshima M, Inoue J (1999) The air-puff evoked escape behavior of the cricket Gryllus bimaculatus and its compensational recovery after cercal ablations. Zool Sci 16: 71-79

Kanou M, Ureshi M, Suematsu Y, Hirao K (2001) Changes in the escape eliciting system of a cricket revealed by sensory deprivation during postembryonic development. Zoo Sci 18: 791796

Kanou M, Teshima N, Nagami T (2002) Rearing conditions required for behavioral compensation after unilateral cercal ablation in the cricket Gryllus bimaculatus. Zool Sci 19: 403-409

Kanou M, Morita S, Matsuura T, Yamaguchi T (2007) Morphology and electrophysiology of water receptors on legs of the cricket, Gryllus bimaculatus. Zool Sci 24: 953-958

Krämer K, Markl H (1978) Flight-inhibition on ground contact in the American cockroach, Periplaneta americana I. Contact receptors and a model for their central connections. J Insect Physiol 24: $577-586$

Matsuura T, Kanou M, Yamaguchi T (2002) Motor program initiation and selection in crickets, with special reference to swimming and flying behavior. J Comp Physiol A 187: 987-995

Murphey RK, Palka J, Hustert R (1977) The cercus-to-giant interneuron system of crickets. II. Response characteristics of two giant interneurons. J Comp Physiol A 119: 285-300
Pflüger HJ, Burrows M (1978) Locusts use the same basic motor pattern in swimming as in jumping and kicking. J Exp Biol. 75: 81-93

Ritzmann RE, Tobias ML, Fourtner CR (1980) Flight activity initiated via giant interneurons of the cockroach: evidence for bifunctional trigger interneurons. Science 210: 443-445

Shiga S, Kogawauchi S, Yasuyama K, Yamaguchi T (1991) Flight behaviour and selective degeneration of flight muscles in the adult cricket (Gryllus bimaculatus). J Exp Biol 155: 661-667

Shimozawa T, Kanou M (1984a) Varieties of filiform hairs: range fractionation by sensory afferents and cercal interneurons of a cricket. J Comp Physiol A 155: 485-493

Shimozawa T, Kanou M (1984b) The aerodynamics and sensory physiology of range fractionation in the cercal filiform sensilla of the cricket Gryllus bimaculatus. J Comp Physiol A 155: 495505

Tauber E, Camhi JM (1995) The wind-evoked escape behavior of the cricket Gryllus bimaculatus: integration of behavioral elements. J Exp Biol 198: 1895-1907

Tomioka K, Yamaguchi T (1980) Steering responses of adult and nymphal crickets to light, with special reference to the head rolling movement. J Insect Physiol 26: 47-57

Wilson DM (1961) The central nervous control of flight in a locust. J Exp Biol 38: 471-490

(Received October 29, 2006 / Accepted June 4, 2007) 